Bull. Austral. Math. Soc.

$11 \mathrm{~F} 46,11 \mathrm{~F} 27$

VOL. 63 (2001) [269-271]

\title{
THE DYADIC TRACE AND ODD WEIGHT COMPUTATIONS FOR SIEGEL MODULAR CUSP FORMS
}

\author{
Cris Poor and David S. Yuen
}

We define the concept of a special positive matrix. We use the dyadic trace to prove the result that $\operatorname{dim} S_{4}^{k}=0$ for odd $k \leqslant 13$ and that $\operatorname{dim} S_{4}^{15} \leqslant 4$.

The computation of $\operatorname{dim} S_{n}^{k}$, the dimension of the space of Siegel modular cusp forms of degree $n$ and weight $k$, may be facilitated by the use of the dyadic trace [5]. Recall the definition of the dyadic trace: for a positive definite $n \times n$ matrix $T$, we define the dyadic trace by $w(T)=\sup \sum_{i} \alpha_{i}$, where the supremum is taken over all dyadic representations $T=\sum_{i} \alpha_{i} \mu_{i}{ }^{t} \mu_{i}$ with $\mu_{i} \in \mathbb{Z}^{n} \backslash\{0\}$ and positive $\alpha_{i} \in \mathbb{R}$.

The following result from [5] gives an explicit finite set of Fourier coefficients that uniquely determine cusp forms of a given weight. Let $f \in S_{n}^{k}$ have Fourier series $f(\Omega)=\sum_{T} a_{T} e(\langle T, \Omega\rangle)$, where the summation is over semi-integral positive definite matrices $T$ (this means $T$ has half integer entries but with integer diagonal entries); the notation is standard, see [1] or [5]. The result is that $f \equiv 0$ if and only if

$$
a_{T}=0 \text { whenever } w(T) \leqslant n \frac{2}{\sqrt{3}} \frac{k}{4 \pi} .
$$

The paper [5] discusses examples for even weights, and this paper addresses the case of odd weights $k$ in $S_{4}^{k}$; namely, we prove the following theorem.

THEOREM. $S_{4}^{k}=0$ for odd $k \leqslant 13$ and $\operatorname{dim} S_{4}^{15} \leqslant 4$.

Proof: Define a positive definite symmetric $n \times n$ matrix $T$ to be special positive if each element of its automorphism group $\operatorname{Aut}_{\mathbb{Z}}(T)$ has determinant 1 . This is a class property. The Fourier coefficients of $f$ satisfy

$$
a_{t_{v} v}=\operatorname{det}(v)^{k} a_{T}
$$

for all $v \in \mathrm{GL}_{n}(\mathbb{Z})\left[2\right.$, p.45]. Note that if $\operatorname{Aut}_{\mathbb{Z}}(T)$ has an element $v$ with determinant -1 , then $k$ odd and $\left({ }^{* *}\right)$ would imply that $a_{T}=0$. Thus for $k$ odd, the support of $f$ consists entirely of special positive $T$.

Received 31st May, 2000

Copyright Clearance Centre, Inc. Serial-fee code: 0004-9727/01 \$A2.00+0.00. 
If $T$ has a 1 on its diagonal, then the lattice corresponding to $T$ has an element of norm 2, and so the reflection in this element's orthogonal hyperplane would stabilise the lattice. Thus such a $T$ would have a reflection in $\operatorname{Aut}_{\mathbf{Z}}(T)$, and so such a $T$ would not be special positive. Table 1 gives an initial list of representatives for all classes of special positive semi-integral $T$ ordered by their dyadic traces. In particular, Table 1 contains all $T$ with $w(T)<6$. Table 1 was constructed using a computer program with Nipp's tables [3] as a database.

\begin{tabular}{|ccccccccccccc|}
\hline$w(T)$ & $16 \operatorname{det} T$ & \# Aut $(T)$ & $T_{11}$ & $T_{22}$ & $T_{33}$ & $T_{44}$ & $2 T_{12}$ & $2 T_{13}$ & $2 T_{23}$ & $2 T_{14}$ & $2 T_{24}$ & $2 T_{34}$ \\
\hline 5 & 105 & 8 & 2 & 2 & 2 & 2 & 2 & 1 & 0 & 0 & 1 & 2 \\
5 & 121 & 24 & 2 & 2 & 2 & 2 & 2 & 1 & 0 & 1 & 1 & 2 \\
5.5 & 145 & 4 & 2 & 2 & 2 & 2 & 2 & 1 & 0 & -1 & -1 & 1 \\
5.5 & 153 & 4 & 2 & 2 & 2 & 2 & 1 & 1 & 0 & 1 & 1 & 2 \\
6 & 161 & 4 & 2 & 2 & 2 & 2 & 2 & 1 & 0 & 0 & 1 & 0 \\
\hline
\end{tabular}

Table 1.

Notice that there are no special positive matrices of dyadic trace less than 5 . By use of $\left(^{*}\right)$, any cusp form $f \in S_{4}^{k}$ of odd weight $k$ would vanish if $4(2 / \sqrt{3})(k / 4 \pi)<5$, which happens if $k<13.61$. This implies that $S_{4}^{k}=0$ for odd $k \leqslant 13$.

For $f \in S_{4}^{15}, f$ is determined by the Fourier coefficients $a_{T}$ for the special positive semi-integral classes $[T]$ with $w(T) \leqslant 4(2 / \sqrt{3})(15 / 4 \pi)$, which implies $w(T) \leqslant 5.52$. Table 1 shows that there are four such classes, which implies that $\operatorname{dim} S_{4}^{15} \leqslant 4$. This completes the proof of the theorem.

The result for $k=11$ is new. The results with $k=13$ and $k=9$ were previously proven in [4] using the techniques of theta series with pluri-harmonics. For $k=17$, the dyadic trace bound turns out to imply $w(T) \leqslant 6.25$. The number of classes of special positive matrices with $w(T) \leqslant 6$ is 15 . This implies $\operatorname{dim} S_{4}^{17} \leqslant 15$; but one might suspect the actual dimension is lower.

\section{REFERENCES}

[1] E. Freitag, Siegelsche modulfunktionen, Grundlehren der Mathematische Wissenschaften 254 (Springer-Verlag, Berlin, Heidelberg, New York, 1983).

[2] H. Klingen, Introductory lectures on Siegel modular forms, Cambridge Studies in Advanced Mathematics 20 (Cambridge University Press, Cambridge, 1990). 
[3] G. Nipp, Quaternary quadratic forms, computer generated tables (Springer-Verlag, Berlin, Heidelbrg, New York).

[4] C. Poor and D. Yuen, 'Dimensions of spaces of Siegel modular forms of low weight in degree four', Bull. Austral. Math. Soc. 54 (1996), 309-315.

[5] C. Poor and D. Yuen, 'Linear dependence among Siegel modular forms', Math. Ann. 318 (2000), 205-234.

Department of Mathematics

Fordham University

Bronx, NY 10458

e-mail: poor@fordham.edu
Math. and Computer Science Department Lake Forest College

555 N. Sheridan Rd.

Lake Forest, IL 60045

e-mail: yuen@lfc.edu 